\title{
Interactive comment on "The competition between fracture nucleation, propagation and coalescence in the crystalline continental upper crust" by Jessica A. McBeck et al.
}

\section{Anonymous Referee \#2}

Received and published: 12 August 2020

The manuscript "The competition between fracture nucleation, propagation and coalescence in the crystalline continental crust" by Jessica A McBeck, Wenlu Zhu, and Francois Renard addresses the controls of development of fracture networks. McBeck et al. present an experimental study in which the fracture network development was assessed via microtomography during triaxial mechanical tests on two dry and one water-saturated sample. The data they acquired is remarkably and the method provides a great example of how fracture networks can be tracked during loading. The main outcome, that stress state and saturated vs dry conditions of the sample are the main controls of which (endmember) fracture network develops on the way to macroscopic failure, is not reflected in the title, introduced, clearly highlighted in the methods, 
represented in the results or adequately discussed. The authors need to address all of the following issues so the community can appreciate the scientific contribution.

Major comments: 1 . The main message of the manuscript is not clear. It is not clear if they want to highlight the methodological approach or the results they obtained by applying the method. The research question for this paper though is well hidden. The scope of the manuscript, the objectives and hypothesis are not clear. 2. It is not clear what the motivation for these experiments was. Data seems to be the same as in previous publications, which is fair to use as getting proposals funded and time allocated to do the experiments can be difficult, but it needs to made clear, where this data is new and where (re)used. 3. In the introduction, the overall concept of how fracture networks develop is not clearly outlined, thus that all assumptions and reasoning is vague. References are missing in many parts, which would allow substantiating some of the party awkward assumptions. The controlling variables which are used in the experiments and seem to be the main outcome are not introduced at all (effect of stress on fracturing, interstitial fluids). 4. The methods do not introduce the techniques applied - both the mechanical loading (e.g. rate of loading) and the tomography (e.g. which voxel size), as well as how you analyse the data (e.g. volume calculation, attribution to which mode). 5. The material used is not introduced at all. No description of the microstructure, no material properties (e.g. porosity). This makes it impossible to relate the tomography images and fracture network development to anything. The nucleation and propagation, especially at lower stress steps will be at grain boundaries and pre-existing defects and flaws. 6. The first part of the results seems to belong to the methods, yet it is not clear which point is made. The description and representation of the results are hard to follow and do not seem to grasp/show important information. For example, you could colour the "new" fractures and the ones that coalescence in the shown steps differently. 7. The structure of each section is flawed. The wording is unclear. Logical jumps make it very hard to follow the text. Especially in the introduction, the methods and results. 8. Most parts of the discussion seem to be about something completely different than the experiment (upscaling- from $10 \mathrm{~mm}$ to upper crustal) or

Interactive comment
Printer-friendly version

Discussion paper 
research question (I am assuming crystalline rocks - yet the discussion is on sedimentary basins). The development of the fracture network is not discussed. References, if given, do not fit the topic. 9. The conclusion is contradicting the introduction in several aspects (e.g. LEFM) and is tedious as it simply repeats some statements made before which are not substantiated in the manuscript. Detailed comments: I have commented on the manuscript in detail for the Abstract and the Introduction (see supplement .pdf). The extent of these comments highlight some of the main issues of the manuscript and are alike for the following sections. The Figures are not fitting the manuscript or provide a visualization to enhance the text, some detailed comments can be found there. In addition but not exclusive for the supplement information comments are: Figure S1: - "vox" $\rightarrow$ "voxel" - The variation in fond size and labelling position is a bit irritating. Could you work on it? - what does this \# refer to? why \#3, \#5 and then \#4. Maybe add to caption what the three panel show. - log scale hardly visible -consider using a different symbol/colour for this type to clearly distinct from the nucleation, above. - Caption: This figure does not show this. It only shows it in comparison to another figure. Please name which figure this relates to. -Caption: The main trends are not indicated (in figure or text) - what are they? To make the point, you could add the trends of the 100 voxels to the figures.

Figure S2: -Again, fond size and labelling position are a bit irritating. Why did you change the colour scheme? -Why is this yield (point) line in red, while in a) they are in the same colour as the other lines

Please also note the supplement to this comment:

https://se.copernicus.org/preprints/se-2020-114/se-2020-114-RC2-supplement.pdf

Interactive comment on Solid Earth Discuss., https://doi.org/10.5194/se-2020-114, 2020. 